\title{
Simple, Rapid, and Highly Efficient Separation of Amino Acid Phenylthiohydantoins by Reversed-Phase High-Performance Liquid Chromatography
}

\author{
Shaun D. Black and Minor J. CoOn \\ Department of Biological Chemistry. Medical School, The University of Michigan. \\ Ann Arbor, Michigan 48109
}

Received October 7, 1981

\begin{abstract}
A rapid and efficient separation of amino acid phenylthiohydantoins by high-performance liquid chromatography has been accomplished by step-gradient elution with use of a mobile phase of acetate-buffered aqueous acetonitrile and an octadecylsilyl stationary phase. Typical analyses are completed in less than $12 \mathrm{~min}$. Peak elution positions in this procedure are highly reproducible (with about $0.2 \%$ variance) and allow unambiguous identification of all residues. A procedure for the optimal positioning phenylthiohydantoin-Arg and-His is given. Molar extinction coefficients at $254 \mathrm{~nm}$, which are particularly useful with common fixed wavelength detectors, are given for 25 amino acid phenylthiohydantoins.
\end{abstract}

The Edman degradation, commonly used in the determination of protein primary structure, results in the sequential conversion of $\mathrm{N}$-terminal amino acid residues to their PTH $^{1}$ derivatives. Although much progress has been made with respect to the refining and automation of his procedure, analysis of the resulting PTH-amino acids has remained inadequate until quite recently. Many high-performance liquid chromatographic separations, both gradient and isocratic, utilizing hydrophobic stationary phases have been reported (1-16). However, a number of problems are seen with these, most notably the lack of adequate resolution. The residues within two groups, PTH-Ser/ PHT-Gln and PTH-Met/PTH-Val/PTHPro, have proven to be the most difficult to separate. The widely varying elution positions of PTH-His and PTH-Arg found by many laboratories have recently been accounted for and controlled (1). Other problems concern lengthy analysis time and the

\footnotetext{
' Abbreviations used: PTH, phenylthiohydantoin; PTC, phenylthiocarbamyl; ODS, octadecylsilane; -Me, methyl ester: TEA, triethylamine; Nle, norleucine.
}

limited consistency of peak retention times, which is most characteristic of gradient elutions. Although it is clear that an isocratic system would be preferable, none described thus far have provided a separation that is rapid and also offers high resolution. As an alternative, we have developed a simple stepgradient system which affords excellent resolution, short analysis time, and easy identification of peaks due to its high degree of reproducibility and quiet baseline.

\section{MATERIALS AND METHODS}

Analyses were performed with an Altex Ultrasphere-ODS column ( $5 \mu \mathrm{m}, 4.6 \times 250$ $\mathrm{mm}$ ) and a Waters liquid chromatograph equipped with a Model 6000A pump, model U6K injector (2.0-ml sample loop), Model 440 absorbance detector set for operation at $254 \mathrm{~nm}$, and Data Module recorder/integrator. The column was jacketed and held at $50^{\circ} \mathrm{C}$ by means of a Haake water recirculating bath.

Elution of the PTH-amino acids was accomplished by means of a step gradient. The 
first solvent, Buffer $\mathrm{A}$, was prepared from $\mathrm{CH}_{3} \mathrm{CN}$ and $1 \mathrm{M}$ stock solutions of acctic acid brought to $\mathrm{pH} 4.5$ with either concentrated $\mathrm{NH}_{3}$ or TEA; ammonium-acetate and TEA-acetate were added to a final acetate concentration of $0.05 \mathrm{M}$ (i.e., $0.0 \mathrm{XM}$ ammonium-acetate $+0.0 \mathrm{Y} \mathrm{M}$ TEA-acetate $=0.05 \mathrm{M})$. The proportion was such that PTH-Arg was positioned between PTH-Thr and PTH-Gly, and PTH-His between PTHAsn and PTH-Ser. An increase in the relative amount of TEA-acetate led to earlier elution of both of these basic PTH derivatives. The ratio of $X: Y$ varied from column to column, but remained constant for at least 500 analyses on a given column; in the present work, $X=4$ and $Y=1$. Buffer A also contained $25 \% \mathrm{CH}_{3} \mathrm{CN}(\mathrm{v} / \mathrm{v})$. Buffer B had a fixed composition of $0.05 \mathrm{M}$ ammoniumacetate and $50 \% \quad \mathrm{CH}_{3} \mathrm{CN}$. Buffers were passed through a $0.5-\mu \mathrm{m}$ Zefluor Teflon filter (Ghia Corporation, Pleasanton, Calif.) and degassed before use.

Samples were prepared in Buffer A. After injection, the injector was returned to the "load" position (thereby removing the 2.0$\mathrm{ml}$ sample loop from the flow path), and the step to Buffer B was made after $0.8 \mathrm{~min}$ had elapsed (flow rate, $1.0 \mathrm{ml} / \mathrm{min}$ ). This caused the interface between the two buffers to appear as a baseline rise $(\Delta A=0.002)$ in the region of PTH-Gly.

Absorption spectra were recorded at ambient temperature with a Varian Cary 219 scanning spectrophotometer with use of $95 \%$ ethanol as solvent and a $1.0-\mathrm{cm}$ pathlength. Extinction coefficients at $254 \mathrm{~nm}$ were calculated from recorded spectra and either $\epsilon_{269}$ (17) or dry weights (as in the case of PTHAsp-Me, PTH-Glu-Me, and PTH-Nle).

High-purity water and $\mathrm{CH}_{3} \mathrm{CN}$ (uv) were purchased from Burdick and Jackson (Muskegon, Mich.), and concentrated ammonia, acetic acid, and TEA from Baker. Triethylamine was treated with Norit, filtered, distilled, and stored under $\mathrm{N}_{2}$ at $-20^{\circ} \mathrm{C}$. PTHamino acids were obtained from Sigma or Mann Research Laboratories, and were used without further purification; high-performance liquid chromatography of these compounds showed them to be quite pure (96.0 $\pm 2.6 \%$ ). The methyl esters of PTH-Asp, PTH-Glu, and PTH-S-carboxymethyl Cys were prepared by treatment of the PTHamino acids with $1 \mathrm{~N}$ methanolic $\mathrm{HCl}$ at $50^{\circ} \mathrm{C}$ for $10 \mathrm{~min}$.

\section{RESULTS AND DISCUSSION}

The chromatographic separation of common PTH-amino acids and other compounds relevant to Edman chemistry $(17,18)$ was studied as shown in Fig. 1. Good resolution with approximately constant peak width was seen throughout the chromatogram; only PTH-Glu and PTH-Asn were poorly resolved. Although minor modifications to the first solvent, Buffer A, could be effected to improve the resolution of this pair $(1,2,5,6)$, the problem was obviated by "conversion" in the presence of methanol (18). This led to formation of the methyl esters of PTHAsp, PTH-Glu, and PTH-S-carboxymethyl Cys, which are adequately resolved.

Reproducibility with this system is excellent. For example, the elution time of PTHNle in 19 serial injections varied by $\pm 1.3 \mathrm{~s}$, or approximately $0.2 \%$. This eliminates the need for "spiking" or the running of frequent standards, and generally affords unambiguous identification of all residues. The baseline stability and high degree of resolution obtained also improve the accuracy of peak integration, especially when the background is high, a situation frequently encountered during the sequence of a large polypeptide. The chromatogram depicted in Fig. 1 was obtained after approximately 500 analyses had been made. The column, under conditions specified, should be good for thousands of chromatographic runs, with little loss in resolution. With PTH-Nle, the limit of detectability was about 2 pmol.

To date, only the isocratic system of Tarr (1) is more rapid. However, resolution in the present system is generally superior, espe- 


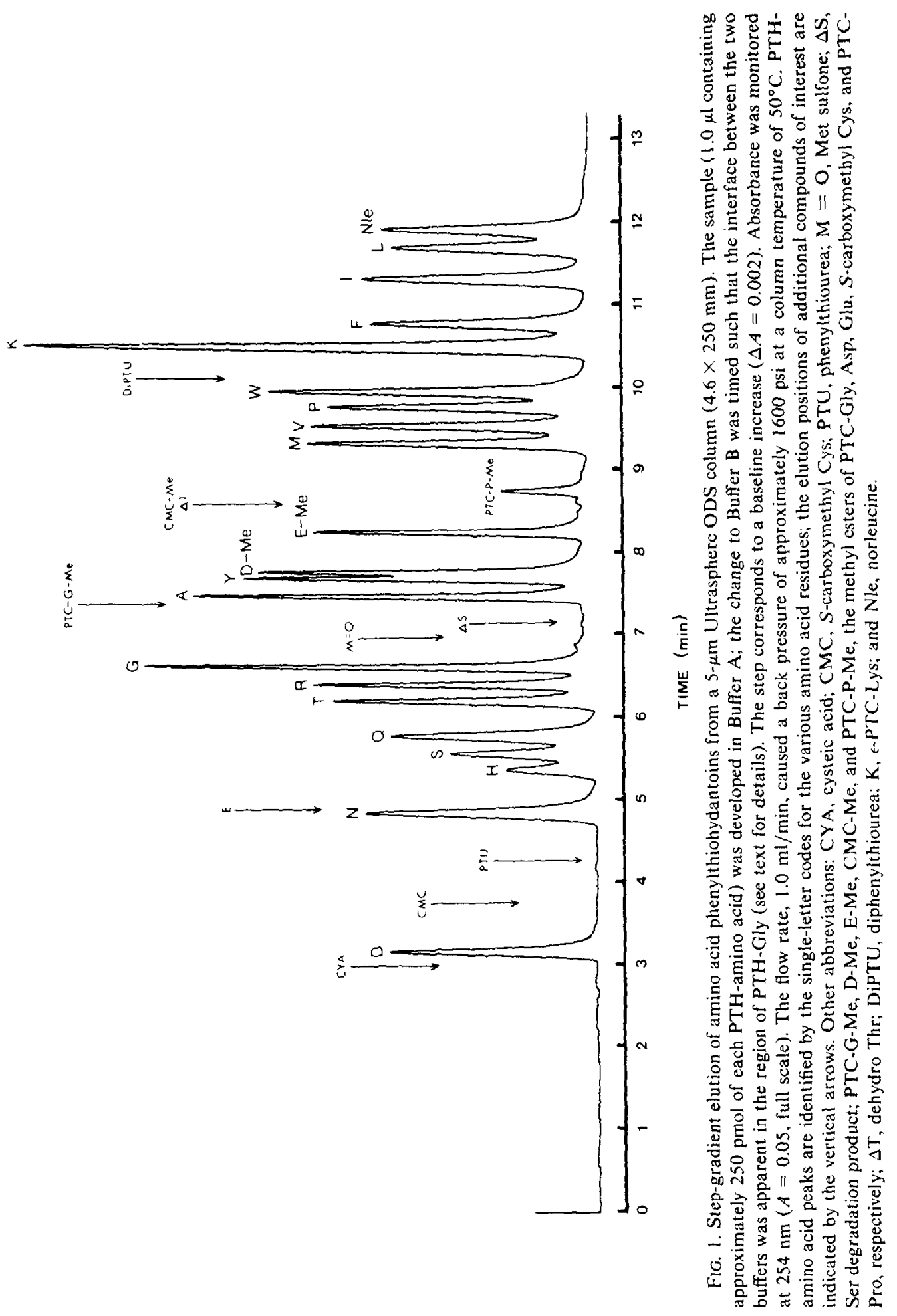


TABLE 1

Physical Properties of amino Acid Phenylthiohydantolns: Molecular WeightS and Molar ABSorptivities

\begin{tabular}{lccc}
\hline \multicolumn{1}{c}{ Compound } & $\begin{array}{c}\text { Molecular } \\
\text { weight }^{a}\end{array}$ & $\begin{array}{c}\epsilon_{254}{ }^{b} \\
\left(\mathrm{M}^{-1} \mathrm{~cm}^{-1}\right)\end{array}$ & $\begin{array}{r}\epsilon_{254} \text { relative to } \\
\text { PTH-Norleucine }\end{array}$ \\
\hline PTH-Alanine & 206.3 & 9,220 & 0.99 \\
PTH-Arginine-HCl & 327.8 & 9,190 & 0.98 \\
PTH-Asparagine & 249.3 & 9,720 & 1.04 \\
PTH-Aspartic acid & 250.3 & 9,190 & 0.98 \\
PTH-Aspartic acid methyl ester & 264.3 & 9,780 & 1.04 \\
PTH-Cysteic acid, Na salt & 308.3 & 9,430 & 1.01 \\
PTH-Glutamic acid & 264.3 & 8,960 & 0.96 \\
PTH-Glutamic acid methyl ester & 278.3 & 10,100 & 1.08 \\
PTH-Glutamine & 263.3 & 9,440 & 1.01 \\
PTII-Glycine & 192.2 & 9,800 & 1.05 \\
PTH-Histidine-HCl & 308.8 & 9,260 & 0.99 \\
PTH-Isoleucine & 248.3 & 9,490 & 1.01 \\
PTH-Leucine & 248.3 & 9,200 & 0.98 \\
PTH-(6-PTC)-Lysine & 398.5 & 11,400 & 1.22 \\
PTH-Methionine & 266.4 & 8,670 & 0.97 \\
PTH-Methionine sulfone & 298.4 & 8,080 & 0.86 \\
PTH-Phenylalanine & 282.4 & 8,390 & 0.90 \\
PTH-Proline & 232.3 & 9,340 & 1.00 \\
PTH-S-Carboxymethylcysteine & 296.5 & 10,100 & 1.08 \\
PTH-Serine & 222.3 & 9,080 & 0.97 \\
PTH-Threonine & 236.3 & 8,830 & 0.94 \\
PTH-Tryptophan & 321.4 & 11,880 & 1.27 \\
PTH-Tyrosine & 298.4 & 8,930 & 0.95 \\
PTH-Valine & 234.3 & 9,140 & 0.98 \\
PTH-Norleucine & 248.3 & 9,360 & $(1.00)$ \\
\hline
\end{tabular}

\footnotetext{
${ }^{a}$ Data taken from Ref. (17).

${ }^{b}$ Values determined from uv absorption spectra relative to $\epsilon_{269}$ given in Ref. (17). PTH-Glu-Me, PTH-Asp-Me, and PTH-Nle values were determined in the present work.
}

cially with respect to the early region of the chromatogram. Phenylthiourea, a ubiquitous by-product of the Edman degradation, is well separated from all PTH-amino acids, which allows the accurate identification and quantitation of the poorly recovered residues PTH-His and PTH-Ser. This is not possible by the method of Tarr. In addition, resolution of PTC-Gly-Me and PTH-Ala prevents the mistaken identification of a glycyl residue as alanine (18).

In practice, the TEA concentration of Buffer A was first adjusted to position PTHArg and PTH-His optimally. High purity of the TEA was found to be essential to keep the baseline rise after the buffer change to a minimum; impure TEA led to large, broad peaks in the latter part of the chromatogram. Next, the timing of the buffer change was adjusted. When the change was made too early, the resolution of PTH-Ser and PTHGln was lost; if it was made too late, resolution of the pairs PTH-Tyr/PTH-Asp-Me and PTH-( $\epsilon$-PTC)-Lys/PTH-Phe was poor.

Most liquid chromatography detectors utilize as their light source the mercury lamp, which has its strongest emission line at $254 \mathrm{~nm}$. Although the absorption maximum of the PTH-amino acids is the region of $269 \mathrm{~nm}$, there is sufficient extinction at $254 \mathrm{~nm}$ to allow sensitive detection. For accurate quantitation, either absolute or rela- 
tive molar extinction coefficients are required. These have been determined for a number of PTH-amino acids with the results shown in Table 1.

The chromatographic separation presented offers the desirable properties of efficient and rapid resolution, sensitivity, reproducibility, and single pump operation. Although this laboratory has implemented the step gradient manually, it is entirely amenable to automated operation. This could be accomplished by the use of a three-way solenoid value at the solvent inlet of the highpressure pump, with control by "timed event outputs" available on many electronic integrators. This system is also attractive because it consumes only $12-14 \mathrm{ml}$ of solvent per analysis; in relation to other methods employing solvent systems of similar composition, this corresponds to 3 to $450 \%$ less buffer per run $(1,9)$. Thus, for a laboratory actively engaged in sequence determination this system offers chromatographic efficiency as well as savings in solvent consumption, instrumentation, and personnel time.

\section{ACKNOWLEDGMENTS}

We wish to thank Drs. G. E. Tarr and A. V. Persson for their useful suggestions and discussion, and Ms. Sharon Pawlowski for technical assistance in the determination of extinction coefficients. This research was supported by Grant AM-10339 from the National Institutes of Health. S.D.B. was the recipient of a Merck Predoctoral Fellowship.

\section{REFERENCES}

1. Tarr, G. E. (1981) Anal. Biochem. 111, 27-32.

2. Lottspeich, F. (1980) Hoppe Seylers Z. Physiol. Chem. 361, 1829-1834.

3. Sottrup-Jensen, L., Petersen, T. E., and Magnusson, S. (1980) Anal. Biochem. 107, 456-460.

4. Fohlman, J., Rask, L., and Peterson, P. A. (1980) Anal. Biochem. 106, 22-26.

5. Harris, J. U., Robinson, D., and Johnson, A. J. (1980) Anal. Biochem. 105, 239-245.

6. Somack, R. (1980) Anal. Biochem. 104, 464-468.

7. Henderson, L. E., Copeland, T. D., and Oroszlan, S. (1980) Anal. Biochem. 102, 1-7.

8. Johnson, N. D., Hunkapiller, M. W., and Hood, L. E. (1979) Anal. Biochem. 100, 335-338.

9. Horn, M. J., Hargrave, P. A., and Wang, J. K. (1979) J. Chromatogr. 180, 111-118.

10. Spatz, R., and Roggendorf, E. (1979) Fresenius $Z$. Anal. Chem. 229, 267-270.

11. Moser, P. W., and Rickli, E. E. (1979) J. Chromatogr. 176, 451-455.

12. Elion, J., Downing, M., and Mann, K. (1978) $J$. Chromatogr. 155, 436-438.

13. Abrahamsson, M., Groningsson, K., and Castensson, S. (1978) J. Chromatogr. 154, 313-317.

14. Margolies, M. N., and Brauer, A. (1978) J. Chromatogr. 148, 429-439.

15. Bhown, A. S., Mole, J. E., Weissinger, A., and Bennett, J. C. (1978) J. Chromatogr. 148, 532535.

16. Zimmerman, C. L., and Pisano, J. J. (1977) in Methods in Enzymology (Hirs, C. H. W., and Timasheff, S., eds.), Vol. 47, pp. 45-51. Academic Press, New York.

17. Edman, P., and Henschen, A. (1975) in Protein Sequence Determination (Needleman, S. B., ed.), 2nd. ed., pp. 233-279, Springer-Verlag, New York/Berlin.

18. Tarr, G. E. (1977) in Methods in Enzymology (Hirs, C. H. W., and Timasheff, S., eds.), Vol. 47, pp. 335-357, Academic Press, New York. 